\title{
Nuclear DNA Content, Base Composition, and Cytogenetic Characterization of Christia obcordata
}

\author{
Hamidou F. Sakhanokho ${ }^{1}$ \\ USDA-ARS, Thad Cochran Southern Horticultural Laboratory, 810 Highway 26 West, Poplarville, \\ MS 39470 \\ Nurul Islam-Faridi \\ U.S. Forest Service, Texas A\&M University, Forest Tree Molecular Cytogenetics Laboratory, \\ Southern Research Station, College Station, TX 77843
}

\begin{abstract}
AdDitional INDEX words. butterfly plant, flow cytometry, fluorescence in situ hybridization, FISH, genome size, ribosomal RNA gene families

ABstract. Christia obcordata is an intriguing small-sized house plant with unusual and attractive features such as its striped leaves. Because very little is known about the plant, we conducted an investigation of its genome and chromosomes. The number of chromosomes was determined using a protoplast technique to prepare root tip chromosome spread and was found to be $2 n=2 x=20$. Flow cytometry was used to determine nuclear DNA content $(1 \mathrm{C}=0.65 \mathrm{pg}=634.4 \mathrm{Mb})$ for $\mathrm{C}$. obcordata and $\mathrm{AT} / \mathrm{GC}$ composition was shown to be $\mathrm{AT} \%=62.8 \% \pm 0.0 \%$ and $\mathrm{GC} \%=$ $37.2 \% \pm 0.0 \%$. Finally, fluorescent in situ hybridization was used to locate ribosomal RNA gene families in $C$. obcordata. Ribosomal RNA gene families, viz. 18S-28S and 5S rDNA, are unique cytomolecular landmarks that provide valuable information about the evolutionary organization of a genome. We have identified one locus each of $18 \mathrm{~S}-28 \mathrm{~S}$ and $5 \mathrm{~S}$ rDNA. The 18S-28S rDNA is located in the subterminal position on the secondary constriction region [also known as the nucleolus organizer region (NOR)] and the $5 \mathrm{~S}$ rDNA is located interstitially close to a centromeric position. The basic information gathered in this study on $C$. obcordata will be helpful in understanding the genetics of this species.
\end{abstract}

The genus Christia belongs to the Fabaceae family and consists of $\approx 13$ species found in tropical Asia and Australia (Flora of China, 2010). The genus Christia has a complicated nomenclature history, which most likely explains the various synonyms under which C. obcordata was listed, including Hedysarum obcordatum, Hedysarum reniforme, Lourea obcordata, Lourea reniforme, and Lourea reniformis (Barham, 1996). Christia obcordata also is known under various common names such as butterfly plant, butterfly leaf, iron butterfly, butterfly stripe, or swallowtail (Culbert, 2007). Leaves are usually trifoliated and have the shape of striped butterfly wings, and both the stems and leaves are slightly hairy (Fig. 1). Like other Christia species, C. obcordata is native to Asia where it can be found in many countries including China, India, Indonesia, Thailand, and Vietnam. The plant is believed to have been introduced from Thailand into the United States, where it is cultivated as an ornamental (Culbert, 2007). There is an increasing interest in C. obcordata because it is such an unusual and eye-catching plant (Fig. 1); therefore, it presents potential for mass production as an ornamental. However, very little is known about the genetics of C. obcordata. For this reason, we decided to determine the chromosome number using a modern protoplast technique to spread root tip chromosomes, nuclear DNA content and base composition using flow cytometry, and the location of ribosomal RNA gene families (18S-28S rDNA and 5S rDNA) using fluorescent in situ hybridization (FISH).

\section{Material and Methods}

Plant material. Plant tissue was excised from greenhousegrown $C$. obcordata plants. In total, five separate plants were

Received for publication 14 Feb. 2013. Accepted for publication 3 Apr. 2013. ${ }^{1}$ Corresponding author. E-mail: Hamidou.Sakhanokho@ars.usda.gov. sampled, and Glycine max 'Polanka' $(2 \mathrm{C}=2.50 \mathrm{pg}$ DNA), kindly supplied by J. Doležel (Experimental Institute of Botany, Botany, Czech Republic), was also grown in a greenhouse and used as an internal standard. Procedures were performed twice on each sample. Flow cytometry analysis was done on a Ploidy Analyzer I/Cell Counter Analyzer (Partec, Münster, Germany) with a mercury lamp (HBO-100; Partec). The flow cytometer had been modified and upgraded with a filter combination (03-340-1000; Partec) adapted to recognize both propidium iodide (PI) and ultraviolet stains depending on its configuration.

4',6-DiAMIdino-2-PHENYLINDOLE STAINING PROCEDURE. A staining kit (Cystain ultraviolet Precise P 05-5002; Partec North America, Swedesboro, NJ) was used for extraction of nuclei and ultraviolet [4',6-diamidino-2-phenylindole (DAPI)] DNA staining. A sample was taken from each of the 10 butterfly plants, trimmed to $\approx 0.5 \mathrm{~cm}^{2}$, and placed in a petri dish. A similar size sample was taken from the G. max 'Polanka' plant and placed in the same petri dish. Both pieces of tissue were covered with $\approx 10$ to 20 drops of extraction buffer (05-5002; Partec North America) from a disposable pipette. The tissue was then diced with a sharp, double-sided razor to produce small slices but without pulverization. Several more drops of extraction buffer were added to the plant and buffer mixture (a total of $400 \mu \mathrm{L}$ or less) and the petri dish was briefly swirled and then incubated for 30 to $60 \mathrm{~s}$. The mixture was washed to the edge of the petri dish, after incubation, with several drops of the staining buffer and filtered through a $50-\mu \mathrm{m}$-mesh filter (CellTrics disposable filter; Partec North America) that was seated on a small test tube. Additional staining buffer was dropped through the filter for a total of no more than $1.6 \mathrm{~mL}$. The test tube was incubated for an additional $1 \mathrm{~min}$ and then analyzed in the flow cytometer set to the blue fluorescence channel. This procedure was performed twice for all samples. 


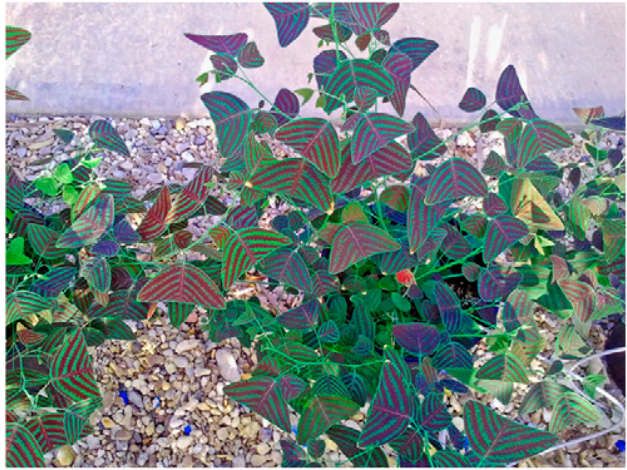

Fig. 1. Mature Christia obcordata plants grown under greenhouse environment at the U.S. Department of Agriculture, Agriculture Research Service unit in Poplarville, MS.

Propidium iodide Staining Procedure. Isolation of nuclei and PI staining was conducted using the same protocol as used for DAPI staining with a few differences as noted subsequently. A staining kit (Cystain PI Absolute P 05-5022; Partec North America) was used for nuclei extraction and PI DNA staining. The kit contained a pre-mixed extraction buffer and the components of the staining solution. The staining solution was prepared on the day of the flow cytometry procedure. The staining recipe was $2 \mathrm{~mL}$ of staining buffer per sample mixed with $12 \mu \mathrm{L}$ of PI solution and $6 \mu \mathrm{L}$ of RNAse solution (055022; Partec North America). The test tube was incubated for an additional 30 to $60 \mathrm{~min}$ and then analyzed in the flow cytometer using green excitation. The flow cytometer produced histograms for each of the sample/standard runs and included means, Cvs, and total cell counts.

NuCLEAR DNA CONTENT, GENOME SIZE, AND BP COMPOSITION. DAPI- and PI-stained nuclei were analyzed using a flow cytometer. Fluorescence ratios, calculated relative to the internal reference $G$. max 'Polanka' $(2 \mathrm{C}=2.50 \mathrm{pg})$, were converted to DNA content values and expressed in picograms following the formula: sample $2 \mathrm{C}$ value (picograms) $=$ reference $2 \mathrm{C}$ value $\times[($ sample $2 \mathrm{C}$ mean peak $) /($ reference $2 \mathrm{C}$ mean peak)]. Conversion from picograms to bp numbers was calculated as follows: genome size $(\mathrm{bps})=\left(0.978 \times 10^{9}\right) \times$ DNA content (picograms) (Doležel et al., 2003; Doležel and Greilhuber, 2010), and the percentage of AT in C. obcordata was measured in relation to the $G$. max standard by comparing the peaks of fluorescence of the DAPI-stained $\mathrm{G}_{0} / \mathrm{G}_{1}$ nuclei following the method described by Godelle et al. (1993): $\mathrm{AT}_{\text {sample }}=\mathrm{AT}_{\text {standard }} \times$ $\left(\mathrm{R}_{\mathrm{DAPI}} / \mathrm{R}_{\mathrm{PI}}\right)^{1 / \mathrm{r}}$, where $\mathrm{AT}_{\text {standard }}(\%)=63.6 \%, \mathrm{R}$ is the ratio of fluorescence intensity from the peak of $C$. obcordata to that of G. $\max$, and $\mathrm{r}$ (binding length) $=3$ (Meister and Barrow, 2007; Portugal and Waring, 1988).

Chromosome Spread preparation. C. obcordata plants were grown in potting medium (Metro-Mix SB-650; Sun Gro Horticulture, Agawam, MA) potting soils in a greenhouse in College Station, TX. Actively growing root tips, $\approx 1 \mathrm{~cm}$, were harvested into $0.8 \%$ (vol/vol) $\alpha$-bromonaphthalene (Sigma, St. Louis, MO) aqueous solution, pre-treated for $2 \mathrm{~h}$ in the dark to accumulate metaphases, and then fixed in 4 ethyl alcohol (95\%):1 glacial acetic acid (Fisher Scientific, Fair Lawn, NJ). The root tips were then enzymatically digested to prepare chromosome spreads as described elsewhere (Jewell and IslamFaridi 1994), except that the enzyme solution was modified as follows: 40\% (vol/vol) Cellulase (C2730; Sigma), 20\% (vol/ vol) Pectinase (P2611; Sigma), 40\% (vol/vol) $0.01 \mathrm{M}$ citrate buffer (trisodium citrate-dihydrate) (Mallinckrodt Baker, Phillipsburg, NJ), citric acid-monohydrate, $\mathrm{pH} 4.5$ (Fisher Scientific), 2\% (wt/vol) Cellulase RS (SERVA Electrophoresis, Heidelberg, Germany), 3\% (wt/vol) Cellulase R10 (Yakult Pharmaceutical, Tokyo, Japan), 1\% (wt/vol) Macerozyme (Yakult Pharmaceutical), and 1.5\% (wt/vol) Pectolyase Y23 (Kyowa Chemical Co., Osaka, Japan).

FluORESCENT IN SITU HYBRIDization. Whole plasmid DNA with an 18S-28S rDNA insert of maize [Zea mays (Zimmer et al., 1988)] or a 5S rDNA insert of sugar beet [Beta vulgaris (Schmidt et al., 1994)] including the spacer region were labeled with biotin-16-dUTP (Biotin-Nick Translation Mix; Roche, Indianapolis, IN) or digoxigenin-11-dUTP (Dig-Nick-Translation Mix; Roche) following the manufacturer's instructions. Agarose gel electrophoresis was used to control the fragment sizes of the probe DNA and labeled nucleotide incorporation was verified by dot-blotting. Briefly, dot blot detection of the labeled probe was performed as follows. The labeled probe $(1.5 \mu \mathrm{L})$ was spotted on a nitrocellulose strip (H-bond membrane; Amersham, Piscataway, NJ), which was dried at $80^{\circ} \mathrm{C}$ for $20 \mathrm{~min}$, rehydrated in buffer I (0.1 M Tris.HCl, pH 7.5; Fisher Scientific), 0.15 M $\mathrm{NaCl}$ (Macron Chemicals, Center Valley, PA) for $1 \mathrm{~min}$, and then incubated in 3\% Bovine Serum Albumin Fraction-V (Roche) in buffer I, and this mixture was referred to as buffer II, at $65^{\circ} \mathrm{C}$ for 45 to $60 \mathrm{~min}$. The strip was dried at $80^{\circ} \mathrm{C}$ for $10 \mathrm{~min}$, rehydrated in buffer II at room temperature for $2 \mathrm{~min}$, and then incubated in $0.1 \mu \mathrm{g} / \mathrm{mm}$ AP conjugated IgG fraction monoclonal mouse antidig (for the dig-labeled probe) or antibiotin (for biotin-labeled probe) (Jackson ImmunoResearch, West Grove, PA) in buffer I for 10 min with gentle agitation, and finally washed in buffer I and buffer III (0.1 M Tris. $\mathrm{HCl}, \mathrm{pH}$ 9; Fisher Scientific), 0.1 M NaCl (Macron Chemicals), and $50 \mathrm{mM} \mathrm{MgCl}_{2}$ (Fisher Scientific) successively for $10 \mathrm{~min}$ in each buffer at room temperature. The strip was then stained in dye solution (BCIP/NBT-Blue Liquid substrate system for membrane; Sigma) for 10 to $20 \mathrm{~min}$ in the dark. The strip was washed in buffer IV (stop buffer, $20 \mathrm{~mm}$ Tris.HCl, pH 7.5; Fisher Scientific), $0.5 \mathrm{~mm}$ EDTA (Fisher Scientific) to stop development, and then dried at $80{ }^{\circ} \mathrm{C}$ for $5 \mathrm{~min}$. The labeled nucleotide incorporation is indicated by dark bright dot/spot on the membrane; in other words, higher intensity is indicative of better incorporation of the labeled nucleotide.

A standard FISH technique was used as previously reported (Hanson et al., 1996; Islam-Faridi et al., 2002, 2009). Sites of biotin-labeled probe hybridization were detected using Cy3conjugated streptavidin (Jackson ImmunoResearch). Sites of digoxigenin-labeled probe binding were visualized using fluorescein-conjugated sheep antidigoxigenin (Roche). Slides were counterstained with DAPI $\left(4 \mu \mathrm{g} \cdot \mathrm{mL}^{-1}\right)$ in McIlvaine buffer [mM citric acid (Fisher Scientific)], $80 \mathrm{~mm} \mathrm{Na} \mathrm{HPO}_{4} \cdot \mathrm{H}_{2} \mathrm{O}$, (Fisher Scientific), $2.5 \mathrm{~mm} \mathrm{MgCl}_{2}$, pH 7.0 (Fisher Scientific), and a small drop $(10 \mu \mathrm{L})$ of Vectashield (Vector Laboratories, Burlingame, CA) was added to the preparation and the slides covered with a glass coverslip to prevent photo-bleaching of the fluorochromes.

Microscopy. Chromosome spreads were viewed under a $63 \times$ plan apo-chromatic objective and digital images were recorded using an epi-fluorescence microscope (Axio Imager M2; Carl Zeiss, Göttingen, Germany) with suitable filter sets (Chroma Technology, Bellows Falls, VT) and a CoolCube 1 
Table 1. Mean values for bp composition, nuclear DNA content, genome size, $\mathrm{CV}$ of $4^{\prime}, 6$-diamidino-2-phenylindole (DAPI)- and propidium iodide (PI)-stained samples, and size standard obtained by flow cytometry analysis of Christia obcordata using Glycine max 'Polanka' $(2 \mathrm{C}=2.50 \mathrm{pg})$ as internal reference standard.

\begin{tabular}{lcr}
\hline Parameter & No. & Mean $\pm \mathrm{sE}^{\mathrm{z}}$ \\
\hline AT content (\%) & 10 & $62.8 \pm 0.0$ \\
GC content (\%) & 10 & $37.2 \pm 0.0$ \\
1C DNA content (pg) & 10 & $0.65 \pm 0.0$ \\
1C Genome size (Mb) & 10 & $634.4 \pm 0.8$ \\
PI-stained C. cordata samples [CV (\%)] & 10 & $2.0 \pm 0.2$ \\
PI-stained G. max samples [CV (\%)] & 10 & $1.9 \pm 0.2$ \\
DAPI-stained C. cordata samples [CV (\%)] & 10 & $4.4 \pm 0.2$ \\
DAPI-stained G. max samples [CV (\%)] & 10 & $3.1 \pm 0.1$
\end{tabular}

${ }^{\mathrm{z}}$ Mean values of 10 runs for five $C$. obcordata and $G$. max mixtures. There were two runs for each C. obcordata and G. max mixture.
(MetaSystems Group, Boston, MA) high-performance chargecoupled device camera. Images were pre-processed with Ikaros and ISIS Version 5.1 (MetaSystems Group) and then further processed with Photoshop CS v8 (Adobe Systems, New York, NY).

\section{Results and Discussion}

Flow CYTOMETRy. We used flow cytometry to determine the nuclear DNA content, genome size, and AT/GC ratio in C. obcordata. A minimum of 3000 nuclei was analyzed per sample. The CV provides information on the quality of the relative fluorescent peaks. In general, cvs below 3\% are considered fully acceptable (Galbraith et al., 1998; Marie and Brown, 1993), but such precision may not be attainable in several "difficult" species for which CVs below 5\% are considered acceptable (Doležel and Bartoš, 2005). In our study, the mean
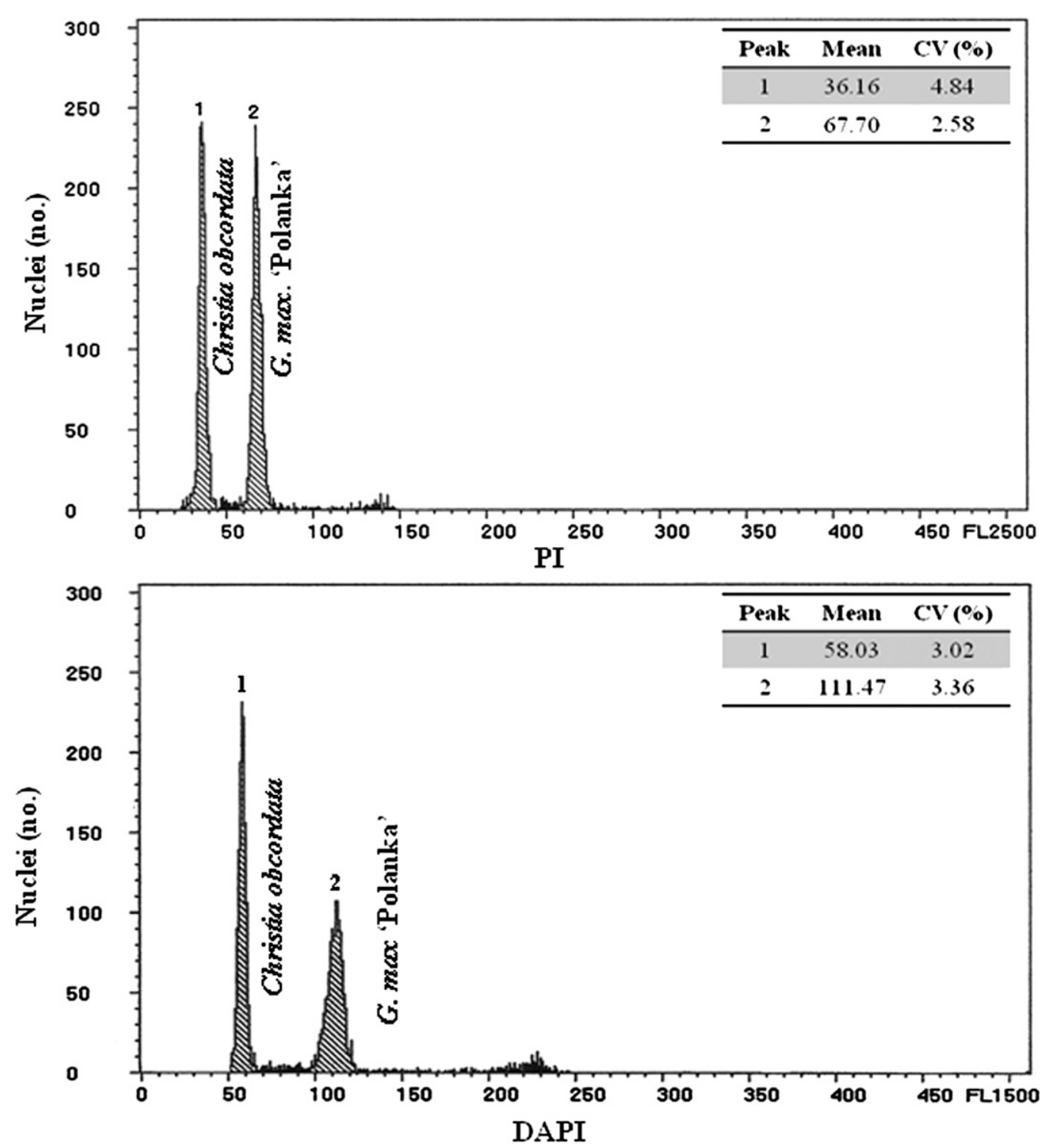

Fig. 2. Histograms of relative nuclear DNA content of Christia obcordata and Glycine max 'Polanka' ( $2 \mathrm{C}=2.50 \mathrm{pg})$ used as internal standard stained with propidium iodide (PI) and 4',6-diamidino-2-phenylindole (DAPI). 
CVs $( \pm$ SE) for DAPI- and PI-stained samples were $4.4 \% \pm 0.2 \%$ and $2.0 \% \pm 0 \%$, respectively (Table 1 ). All cV values for both PI and DAPI were well within the range defined by Doležel and Bartoš (2005).

Nuclear DNA content and genome size for $C$. obcordata were determined for the first time and found to be $1 \mathrm{C}=0.65 \mathrm{pg}=$ 634.4 Mb (Table 1). Genome size in plants varies nearly 2000fold from $63 \mathrm{Mb}$ in Genlisea margaretae to $124,852 \mathrm{Mb}$ in Fritillaria assyriaca (Greilhuber et al., 2006; Leitch and Bennett, 2007), so the genome of $C$. obcordata is $\approx 10$ times the size of that of the species with the smallest genome, namely $G$. margaretae. To the best of our knowledge, this is the first report of genome size determination in any Christia species, so comparison of our results with genome sizes of other Christia species is not currently possible. Knowledge of the genome size of a given plant species is important for many reasons, ranging from guiding sequencing purposes to methods of fingerprinting and determination of the number of clones needed for complete genome coverage in a genomic library or the establishment of efficient insertional mutagenesis strategies in a large-scale genomic studies (Leitch and Bennett, 2007; Peer et al., 2003). We also determined AT/GC composition using flow cytometry and $G$. max 'Polanka' as the standard and found that $\mathrm{AT} \%=$ $62.8 \% \pm 0.0 \%$ and $\mathrm{GC} \%=37.2 \% \pm 0.0 \%$ in C. obcordata (Fig. 2; Table 1). Although base composition is not as an important taxonomic criterion in plants as it is in microorganisms, it still provides valuable information, which is useful in characterizing plant species and is sometimes a useful parameter in identifying speciation relationships or heterochromatin evolution patterns (Godelle et al., 1993; Leitch and Bennett, 2007).

Chromosome spread. We successfully used the protoplast technique to obtain high-quality mitotic metaphase (somatic) chromosome spreads from root tip meristems of $C$. obcordata. The spreads were mostly free of cell walls, nuclear membranes, and cytoplasmic debris, and the chromosome morphology was excellent, allowing for clear visualization of non-distorted chromosomes. All chromosomes of $C$. obcordata could easily be separated from each other with essentially no overlap (Fig. 3). Well-separated prometaphase and metaphase chromosomes that are free of cell walls, nuclear membranes and cytoplasmic debris are prerequisites for in situ hybridization, and these results were obtained by successfully preparing $C$. obocordata root tip chromosomes without squashing the meristematic tissue under a glass coverslip as described by Jewell and Islam-Faridi (1994). The chromosomes are mostly metacentric with highly heterochromatic pericentromeric regions and euchromatic distal portions of each chromosome containing the expressed DNA as revealed by dark and light Azure blue staining, respectively.

A chromosome number of $2 n=2 x=22$ has been reported in a Christia species before, namely $C$. vespertilionis, commonly referred to as red butterfly wing (Goldblatt, 1981), but to our knowledge, the present study is the first report for C. obcordata. In this study, we obtained results showing a chromosome number of $2 n=2 x=20$ for $C$. obcordata (Fig. 3), suggesting that there are at least two basic chromosome numbers ( $x=10$ and $x=11)$ within the genus Christia.

FluORESCENT IN SITU hYbRidization. FISH can be instrumental in the study of important details of the structural organization of a given plant genome. Moreover, ribosomal RNA gene families (18S-28S rDNA, 5S rDNA) provide valuable cytological landmarks for karyotyping and studying the relationships between species and genera. For example, Jiang and Gill (1994) demonstrated that the location of the 18S-26S rRNA genes provided excellent landmarks for investigating the evolution of polyploid wheats (Triticum aestivum). Furthermore, detection of changes in positions of rDNA sites may be useful in identifying small chromosomal rearrangements (Castilho and Heslop-Harrsion, 1995), which could play a role in speciation as suggested by a study of rDNA FISH pattern between two Pinus subgenera (Cai et al., 2006). Indeed, a multitude of studies of rDNA variation and structural positions have been conducted to provide an evolutionary and phylogenetic view of many plant species, including Arabidopsis species [A. thaliana, A. wallichii, A. pumila, A. griffithiana (Maluszynska and Heslop-Harrison, 1993)], Gossypium hirsutum (Hanson et al., 1996), and 15 wild diploid Coffea species and cultivated tetraploid Coffea arabica (Hamon et al., 2009). One site each of $18 \mathrm{~S}-28 \mathrm{~S}$ and $5 \mathrm{~S}$ rDNA has been identified in C. obocordata (Fig. 4). The 18S-28S rDNA is located in the subterminal position on the secondary constriction region (also known as NOR) and the 5S rDNA site is located interstitially close to a centromeric position of a pair of homologous chromosomes. Almost no sign of DAPI stain was observed in the NOR region, which is

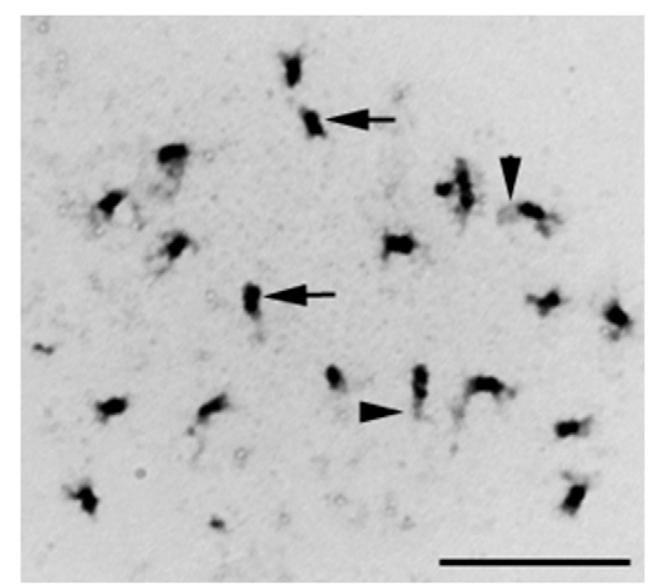

Fig. 3. Somatic chromosome $(2 n=2 x=20)$ spread (late prophase-early prometaphase stage) of Christia obcordata stained with $0.2 \%$ azure blue (A4043; Sigma, St. Louis, MO) and made permanent with a drop of Euparol (Fisher Scientific, Fair Lawn, NJ). The pericentromeric regions are highly heterochromatic (arrows) and the distal portions of each chromosome are euchromatic (stained lightly, arrowheads) that contained the expressed DNA; bar $=10 \mu \mathrm{m}$.
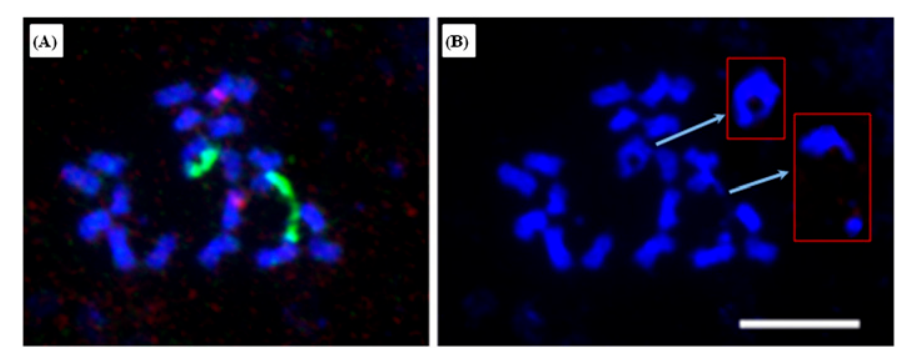

Fig. 4. Fluorescent in situ hybridization (FISH) probed with $18 \mathrm{~S}-28 \mathrm{~S}$ rDNA (green signals) and 5S rDNA (red signals) clones on somatic chromosome spread of Christia obcordata (A) and 4',6-diamidino-2-phenylindole (DAPI)stained chromosomes of the same spread (B). Insets in (B) show larger images (arrows) of the satellited chromosomes that contained the 18S-28S rDNA locus; bar $=5 \mu \mathrm{m}$. 
high in GC content but low in AT, and DAPI binds preferentially and strongly to AT bases (Wilson et al., 1989). The NOR is observed to be stretched as revealed by the FISH signal (Fig. 4).

From an aesthetic and ornamental point of view, $C$. obcordata has the potential for wider adoption as a house plant because of its size and unusual and unique features, particularly its striped leaves (Fig. 1). Unfortunately, not much scientific work has been done on this species to answer some basic questions about the plant. For example, anecdotal reports by some homeowners often paint conflicting pictures on its growth habits and requirements. From a practical point, to intelligently outline a program for improving a variety, the cytogenetical behavior of the species must be understood (Myers and Hill, 1940). To that end, the results on chromosome number, nuclear DNA content, genome size, base composition as well as ribosomal RNA gene families that we presented here will contribute greatly to our understanding of the genetics of the genus Christia in general and, in particular, C. obcordata.

\section{Literature Cited}

Barham, J.M. 1996. Christia vespertilionis var. verspertilionis Leguminose. Curtis's Bot. Mag. 13:19-21.

Cai, Q., D. Zhang, Z.-L. Liu, and X.-R. Wang. 2006. Chromosomal localization of $5 \mathrm{~S}$ and $18 \mathrm{~S}$ rDNA in five species of subgenus Strobus and their implications for genome evolution of Pinus. Ann. Bot. (Lond.) 97:715-722.

Castilho, A. and J.S. Heslop-Harrison. 1995. Physical mapping of 5S and 18S-25S rDNA and repetitive DNA sequences in Aegilops umbellulata. Genome 38:91-96.

Culbert, D. 2007. Stripe: New wings for the home. 14 Sept. 2012.

$<$ http://okeechobee.ifas.ufl.edu/News\%20columns/Stripe.htm>.

Doležel, J. and J. Bartoš. 2005. Plant DNA flow cytometry and estimation of nuclear genome size. Ann. Bot. (Lond.) 95:99-110.

Doležel, J., J. Bartoš, H. Voglmayr, and J. Greilhuber. 2003. Nuclear DNA content and genome size of trout and human. Cytometry 51:127-128.

Doležel, J. and J. Greilhuber. 2010. Nuclear genome size: Are we getting closer? Cytometry A 77:635-642.

Flora of China. 2010. 129. Christia Moench, Suppl. Meth. 39. 1802. Flora of China 10:289-290. 15 Sept. 2012.<http://flora.huh.harvard. edu/china/pdf/PDF10/Christia.pdf>.

Galbraith, D.W., G.M. Lambert, J. Macas, and J. Doležel. 1998. Analysis of nuclear DNA content and ploidy in higher plants, p. 7.6.1-7.6.22. In: Robinson, J.P., Z. Darzynkiewicz, P.N. Dean, L.G. Dressler, A. Orfao, P.S. Rabinovitch, C.C. Stewart, H.J. Tanke, and L.L. Wheeless (eds.). Current protocols in cytometry. Wiley, New York, NY.

Godelle, B., D. Cartier, D. Marie, S.C. Brown, and S. Siljak-Yakovlev. 1993. Heterochromatin study demonstrating the non-linearity of fluorometry useful for calculating genomic base-composition. Cytometry 14:618-626.

Goldblatt, P. 1981. Chromosome numbers in legumes II. Ann. Mo. Bot. Gard. 68:551-557.

Greilhuber, J., T. Borsch, K. Müller, A. Worberg, S. Porembski, and W. Barthlott. 2006. Smallest angiosperm genomes found in Lentibulariaceae, with chromosomes of bacterial size. Plant Biol. 8:770777.

Hamon, P., S. Siljak-Yakovlev, S. Srisuwan, O. Robin, V. Poncet, S. Hamon, and A. de Kochko. 2009. Physical mapping of rDNA and heterochromatin in chromosomes of 16 Coffea species: A revised view of species differentiation. Chromosome Res. 17:291304.

Hanson, R.E., M.N. Islam-Faridi, E.A. Percival, C.F. Crane, T.D. McKnight, D.M. Stelly, and H.J. Price. 1996. Distribution of 5S and $18 \mathrm{~S}-28 \mathrm{~S}$ rDNA loci in a tetraploid cotton (Gossypium hirsutum L.) and its putative diploid ancestors. Chromosoma 105:55-61.

Islam-Faridi, M.N., K.L. Childs, P.E. Klein, G. Hodnett, M.A. Menz, R.R. Klein, J. Mullet, D.M. Stelly, and H.J. Price. 2002. A molecular cytogenetic map of sorghum chromosome 1: Fluorescence in situ hybridization analysis with mapped bacterial artificial chromosomes. Genetics 161:345-353.

Islam-Faridi, M.N., C.D. Nelson, S.P. DiFazio, L.E. Gunter, and G.A. Tuskan. 2009. Cytogenetic analysis of Populus trichocarpaRibosomal DNA, telomere repeat sequence, and marker-selected BACs. Cytogenet. Genome Res. 125:74-80.

Jewell, D.C. and M.N. Islam-Faridi. 1994. Details of a technique for somatic chromosome preparation and C-banding of maize, p. 484-493. In: Freeling, M. and V. Walbot (eds.). The maize handbook. SpringerVerlag, New York, NY.

Jiang, J. and B.S. Gill. 1994. New 18S.26S ribosomal RNA gene loci: Chromosomal landmarks for the evolution of polyploid wheats. Chromosoma 103:179-185.

Leitch, I.J. and M.D. Bennett. 2007. Genome size and its uses: Impact of flow cytometry, p. 153-176. In: Doležel, J., J. Greilhuber, and J. Suda (eds.). Flow cytometry with plant cells: Analysis of genes, chromosomes and genomes. Wiley-VCH, Weinheim, Germany.

Maluszynska, J. and J.S. Heslop-Harrison. 1993. Molecular cytogenetics of the genus Arabidopsis: In situ localization of rDNA sites, chromosome numbers and diversity in centromeric heterochromatin. Ann. Bot. (Lond.) 71:479-484.

Marie, D. and S.C. Brown. 1993. A cytometric exercise in plant DNA histograms, with 2C values for 70 species. Biol. Cell 78:41-51.

Meister, A. and M. Barrow. 2007. DNA base composition of plant genomes, p. 177-185. In: Doležel, J., J. Greilhuber, and J. Suda (eds.). Flow cytometry with plant cells: Analysis of genes, chromosomes and genomes. Wiley-VCH, Weinheim, Germany.

Myers, W.M. and H.D. Hill. 1940. Studies of chromosomal association and behavior and occurrence of aneuploidy in autotetraploid grass species, orchard grass, tall oat grass, and crested wheatgrass. Bot. Gaz. 102:236-255.

Peer, W.A., M. Mamoudian, B. Lahmer, B.D. Reeves, A.S. Murphy, and D.E. Salt. 2003. Identifying model metal hyperaccumulating plants: Germplasm analysis of 20 Brassicaceae accessions from a wide geographical area. New Physiol. 159:421-430.

Portugal, J. and M.J. Waring. 1988. Assignment of DNA binding sites for 4',6-diamidino-2-phenylindole and bisbenzimide (Hoechst 33258). A comparative footprinting study. Biochim. Biophys. Acta 949:158-168.

Schmidt, T., T. Schwarzacher, and J.S. Heslop-Harrison. 1994. Physical mapping of rRNA genes by fluorescent in situ hybridization and structural analysis of 5S rRNA genes and intergenic spacer sequences in sugar-beet (Beta vulgaris L.). Theor. Appl. Genet. 88:629-636.

Wilson, W.D., F.A. Tanious, H.J. Barton, R.L. Jones, L. Strekowski, and D.W. Boykin. 1989. Binding of 4',6-diamidino-2-phenylindole (DAPI) to GC and mixed sequences in DNA: Intercalation of a classical groove-binding molecule. J. Amer. Chem. Soc. 111:50085010 .

Zimmer, E.A., E.R. Jupe, and V. Walbot. 1988. Ribosomal gene structure, variation and inheritance in maize and its ancestors. Genetics 120:1125-1136. 